\title{
Little known Orphninae (Coleoptera, Scarabaeidae) collected by the Madagascan expeditions of the California Academy of Sciences
}

\author{
Andrey V. Frolov ${ }^{\dagger}$ \\ † Zoological Institute of Russian Academy of Sciences, Sankt-Petersburg, Russia
}

Corresponding author: Andrey V. Frolov (aphodius@rambler.ru)

Academic editor: Lyubomir Penev

Received: 30 Oct 2013 | Accepted: 20 Nov 2013 | Published: 27 Nov 2013

Citation: Frolov A (2013) Little known Orphninae (Coleoptera, Scarabaeidae) collected by the Madagascan expeditions of the California Academy of Sciences. Biodiversity Data Journal 1: e1016. doi: $10.3897 /$ BDJ.1.e1016

\section{Abstract}

New locality records for Madecorphnus niger Frolov, 2010, M. falcatus Paulian, 1992, M. simplex Frolov, 2010, and Triodontus itremoi Paulian, 1977, are given. Endophallus armature of M. niger Frolov, 2010, is described and illustrated.

\section{Keywords}

Scarab beetles; orphnines; new species; Madecorphnus; Triodontus; Madagascar

\section{Introduction}

Madagascar houses a rich and taxonomically diverse fauna of the scarab beetles of subfamily Orphninae. Thirty five species of four genera are known and all of them are endemic to the island (Frolov 2010, Frolov and Montreuil 2009, Paulian 1977, Paulian 1992). Only three species (Triodontus nitidulus (Guérin-Méneville, 1844), Pseudorphnus coquerelii (Fairmaire, 1868), and Renorphnus clementi (Petrovitz, 1971)) have a reasonably wide distribution and are known from a large number of specimens, which 
might indicate their adaptation to feeding on crops (documented for $T$. nitidulus larvae: Randriamanantsoa et al. (2010). All other species are rare in collections and many are known from type specimens only. Distribution of taxa is biased towards indigenous forests of the central domain of the Eastern Region and many species ranges may now be limited to isolated patches of forests.

In 2000s, researchers from the California Academy of Sciences (San-Francisco, USA) undertook insect surveys on Madagascar which yielded a rich orphnine material including a few new species (Frolov 2011, Frolov 2012). However, a part of this material, kept in vials with alcohol, was not available for study until recently. In this material, a few rare Madagascan orphnine species were identified. New locality records for these species are given in the present communication. The endophallus armature of $M$. niger Frolov, 2010, is described and illustrated.

\section{Materials and methods}

All examined material is housed in the collection of California Academy of Sciences (SanFrancisco, USA).

Preparation of genitalia follows the common technique used in entomological research. The photograph was taken with a Leica MZ9.5 stereo microscope and a Leica DFC290 digital camera from specimen in glycerol. Partially focused serial images were combined in Helicon Focus software (Helicon Soft Ltd.) to produce completely focused image. The photograph was not altered except for levels and tone correction in Adobe Photoshop (Adobe Inc.). The distribution map was generated with ArcGIS software. As the base map, a Madagascar vegetation map (CEPF Madagascar Vegetation Mapping Project of Royal Botanic Gardens, Kew, Missouri Botanical Garden, and Conservation International's Center for Applied Biodiversity Science; http://www.vegmad.org) was used. Co-ordinates of the localities were taken from the specimen labels.

\section{Taxon treatments}

\section{Madecorphnus niger Frolov, 2010}

\section{Materials}

a. scientificName: Madecorphnus niger, country: Madagascar; stateProvince: Antananarivo; verbatimLocality: $3 \mathrm{~km} 41^{\circ} \mathrm{NE}$ Andranomay, $11.5 \mathrm{~km} 147^{\circ} \mathrm{SSE}$ Anjozorobe; verbatimElevation: $1300 \mathrm{~m}$; verbatimLatitude: $18^{\circ} 28^{\prime} 24^{\prime \prime S}$; verbatimLongitude: $47^{\circ} 57^{\prime} 36^{\prime \prime} \mathrm{E}$; eventDate: 5-13 Dec 2000; individualCount: 1; sex: male; recordedBy: B. Fisher; identifiedBy: Andrey V. Frolov; collectionID: urn:Isid:biocol.org:col:35143; institutionCode: CAS; collectionCode: CASENT

b. scientificName: Madecorphnus niger, country: Madagascar; verbatimLocality: Toamasina Parc National de Zahamena, Onibe River; verbatimElevation: $780 \mathrm{~m}$; verbatimLatitude: $17^{\circ} 45^{\prime} 33^{\prime \prime S}$; verbatimLongitude: $048^{\circ} 51^{\prime} 17 " \mathrm{E}$; eventDate: $21-23$ February; individualCount: 1; sex: male; recordedBy: B. Fisher; identifiedBy: Andrey V. Frolov; 
collectionID: urn:Isid:biocol.org:col:35143; institutionCode: CAS; collectionCode: CASENT

\section{Distribution}

This species was known from 2 types from Perinet (Andasibe). The two additional localities reported here are situated $70 \mathrm{~km} \mathrm{NNW}$ and $140 \mathrm{~km}$ NNE of the type locality, both within the humid forest biome (Fig. 3).

\section{Taxon discussion}

Type specimens of $M$. niger were damaged (lacked endophalluses) and the species was described chiefly on the basis of the distinctive shape of the aedeagus. Examination of the newly available material showed that the endophallus armature is also quite distinctive. The armature consists of three similar, highly sclerotized, toothlike sclerites (Fig. 1). Other sclerites or fields of spinules are absent.

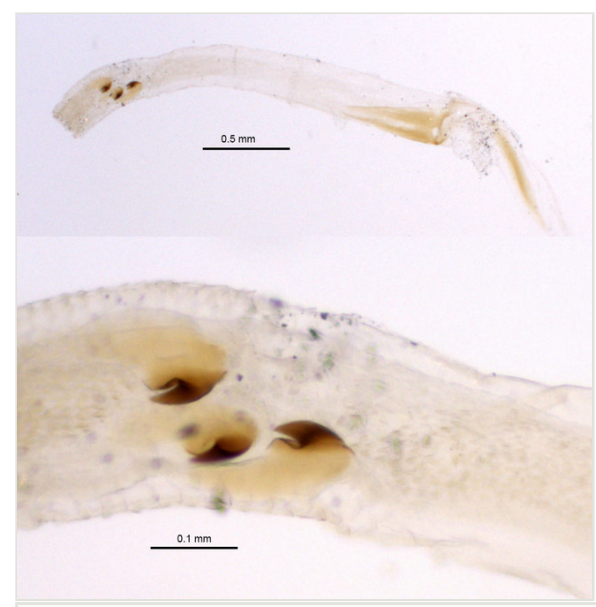

Figure 1.

Madecorphnus niger Frolov, endophallus armature.

\section{Madecorphnus falcatus Paulian, 1992}

\section{Material}

a. scientificName: Madecorphnus falcatus; genus: Madecorphnus; specificEpithet: falcatus; scientificNameAuthorship: Paulian, 1992; country: Madagascar; verbatimLocality: Toamasina, Parcelle K9 Tampolo; verbatimElevation: $10 \mathrm{~m}$; verbatimLatitude: $17^{\circ} 10^{\prime} 30^{\prime \prime S}$; verbatimLongitude: 49¹6'05"E; eventDate: 19 April 2004; individualCount: 1; sex: male; recordedBy: California Acad. of Sciences ant team; identifiedBy: Andrey V. Frolov; collectionID: urn:Isid:biocol.org:col:35143; institutionCode: CAS; collectionCode: CASENT 


\section{Distribution}

This species was known from the only type specimen collected in Antanambe, on the eastern coast of Madagascar. The additional specimen (Fig. 2) was collected some 100 $\mathrm{km}$ south (Fig. 3), in littoral forest litter. This species may be a littoral forest specialist.

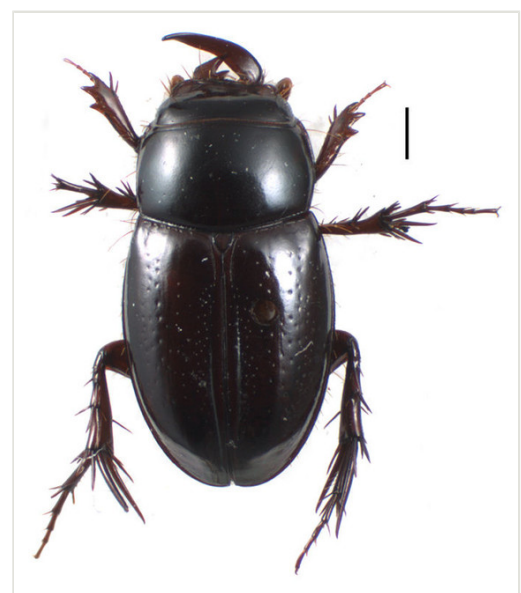

Figure 2.

Madecorphnus falcatus Paulian, male, habitus.

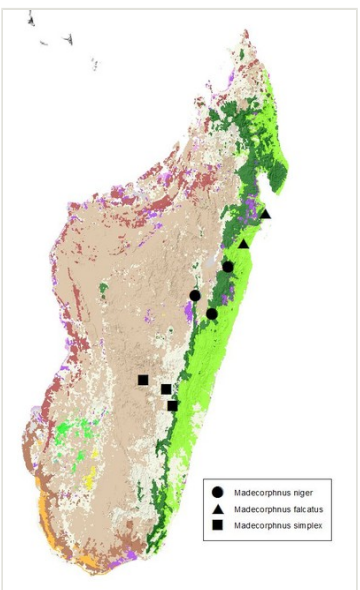

Figure 3.

Madecorphnus spp. Locality map. 


\section{Madecorphnus simplex Frolov, 2010}

\section{Materials}

a. scientificName: Madecorphnus simplex; genus: Madecorphnus; specificEpithet: simplex; scientificNameAuthorship: Frolov, 2010; country: Madagascar; stateProvince:

Fianarantsoa; verbatimLocality: Parc National Ranomafana, at broken bridge; verbatimElevation: $1110 \mathrm{~m}$; verbatimLatitude: 17¹0'30"S; verbatimLongitude: $49^{\circ}$ 16'05"E; eventDate: 19-26 March 2002; individualCount: 1; sex: male; recordedBy: R. Harin'Hala; identifiedBy: Andrey V. Frolov; collectionID: urn:Isid:biocol.org:col:35143; institutionCode: CAS; collectionCode: CASENT

b. scientificName: Madecorphnus simplex; genus: Madecorphnus; specificEpithet: simplex; scientificNameAuthorship: Frolov, 2010; country: Madagascar; stateProvince:

Fianarantsoa; verbatimLocality: Foret d'Atsirakambiaty, $7.6 \mathrm{~km} 285^{\circ} \mathrm{WNW}$ Itremo; verbatimElevation: $1550 \mathrm{~m}$; verbatimLatitude: $20^{\circ} 35^{\prime} 36^{\prime \prime S}$; verbatimLongitude: $046^{\circ}$ 33'48"E; eventDate: 22-26 January 2003; individualCount: 1; sex: male; recordedBy: Fisher, Griswold et al.; identifiedBy: Andrey V. Frolov; collectionID: urn:Isid:biocol.org:col:35143; institutionCode: CAS; collectionCode: CASENT

\section{Distribution}

The species was described from one specimen from Ambatofitorahana. The new locality records include Itremo Massif and Ranomafana National Park (Fig. 3).

\section{Triodontus itremoi Paulian, 1977}

\section{Materials}

a. scientificName: Triodontus itremo; genus: Triodontus; specificEpithet: itremoi; scientificNameAuthorship: Paulian, 1977; country: Madagascar; verbatimLocality: Foret d'Atsirakambiaty, $7.6 \mathrm{~km}$ 285TA WNW Itremo; verbatimElevation: $1550 \mathrm{~m}$; verbatimLatitude: $20^{\circ} 35^{\prime} 36^{\prime \prime S}$; verbatimLongitude: $46^{\circ} 33^{\prime} 48^{\prime \prime E}$; eventDate: $22-26$ January 2003; individualCount: 6 males and 10 females; recordedBy: Fisher, Griswold et al.; identifiedBy: Andrey V. Frolov; collectionID: urn:Isid:biocol.org:col:35143; institutionCode: CAS; collectionCode: CASENT

b. scientificName: Triodontus itremo; genus: Triodontus; specificEpithet: itremoi; scientificNameAuthorship: Paulian, 1977; country: Madagascar; verbatimLocality: Toamasina Ambatovy, 12.4 km NE Moramanga; verbatimElevation: 1040 m; verbatimLatitude: $18^{\circ} 51^{\prime} 29^{\prime \prime} \mathrm{S}$; verbatimLongitude: 048 $17^{\circ} 06^{\prime \prime} \mathrm{E}$; eventDate: 5-8 March 2007; individualCount: 4 males and 2 females; recordedBy: B.L.Fisher et al.; identifiedBy: Andrey V. Frolov; collectionID: urn:Isid:biocol.org:col:35143; institutionCode: CAS; collectionCode: CASENT

c. scientificName: Triodontus itremoi; genus: Triodontus; specificEpithet: itremoi; scientificNameAuthorship: Paulian, 1977; country: Madagascar; verbatimLocality: Toamasina Ambatovy, 12.4 km NE Moramanga; verbatimLatitude: $18^{\circ} 50^{\prime 22}$ " S; verbatimLongitude: 048 $18^{\prime} 30^{\prime \prime}$ E; eventDate: 4-7 March 2007; individualCount: 1 male; recordedBy: B.L.Fisher et al.; identifiedBy: Andrey V. Frolov; collectionID: urn:Isid:biocol.org:col:35143; institutionCode: CAS; collectionCode: CASENT 


\section{Distribution}

This species was described from two rather distant localities, in the Itremo Massif and Tsiroanomandidy (Bongolava district). New records extend the known species range to the eastern slopes of the central plateau occupied by the largest remnants of rain forest (Fig. 4).

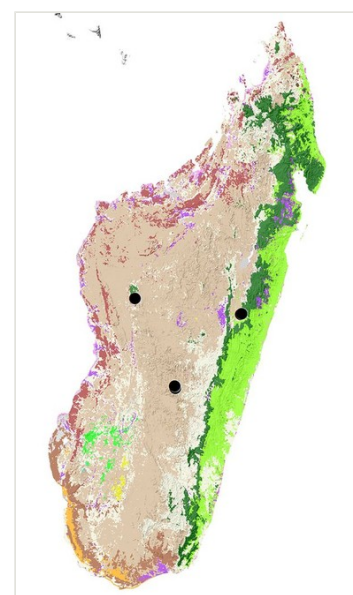

Figure 4.

Triodontus itremoi. Locality map.

\section{Acknowledgements}

I am thankful to Dave Kavanaugh, Jere Schweikert, Igor Sokolov, and Norm Penny for help during my visit to California Academy of Sciences. This work was supported by the Ernst Mayr Travel Grant and, partly, by Russian Foundation for Basic Research (grant 13-04-01002-a).

\section{References}

- Frolov AV (2010) Revision of the Madagascan genus Madecorphnus Paulian (Coleoptera, Scarabaeidae, Orphninae). Journal of Natural History 44: 1095-1111. [In English]. DOI: 10.1080/00222931003632765

- Frolov AV (2011) A new species of the Madagascan genus Pseudorphnus (Coleoptera: Scarabaeidae: Orphninae). Zootaxa 2836: 65-68. URL: http://www.mapress.com/zootaxa/2011/f/ z02836p068f.pdf

- Frolov AV (2012) Three new species of scarab beetle genus Madecorphnus Paulian, 1992 (Coleoptera: Scarabaeidae: Orphninae) from Madagascar. Proceedings of the Zoological Institute RAS 316: 159-165. [In English]. URL: http://www.zin.ru/journals/trudyzin/doc/vol $3162 /$ TZ_316_2_Frolov.pdf 
- Frolov AV, Montreuil O (2009) A new genus of Orphninae (Coleoptera, Scarabaeidae) from Madagascar. Zoosystematica Rossica 18: 65-69. [In English]. URL: http://www.zin.ru/animalia/ coleoptera/pdf/frolov_montreuil_2009.pdf

- Paulian R (1977) Les Orphnidae de Madagascar. Bulletin du Muséum National d'histoire naturelle, Paris. Ser. 3. 411: 1199-1223. [In French].

- Paulian R (1992) Un nouveau genre et une nouvelle espèce d'Orphnidae de Madagascar (Coleoptera, Scarabaeoidea). Revue Française d'Entomologie (N.S.) 14: 169-171. [In French].

- Randriamanantsoa R, Ralisoa OB, Ratnadass A, Vercambre B (2010) Les larves des Scarabaeoidea des régions de haute et moyenne altitudes du Centre de Madagascar. Zoosystema 32: 19-72. [In French]. DOI: 10.5252/z2010n1a2 